\title{
Linear Discrepancy of Chain Products and Posets with Bounded Degree
}

\author{
Jeong-Ok Choi*, Kevin G. Milans ${ }^{\dagger}$, and Douglas B. West ${ }^{\ddagger}$
}

\begin{abstract}
The linear discrepancy of a poset $P$, denoted $\operatorname{ld}(P)$, is the minimum, over all linear extensions $L$, of the maximum distance in $L$ between two elements incomparable in $P$. With $r$ denoting the maximum vertex degree in the incomparability graph of $P$, we prove that $\operatorname{ld}(P) \leq\lfloor(3 r-1) / 2\rfloor$ when $P$ has width 2 . Tanenbaum, Trenk, and Fishburn asked whether this upper bound holds for all posets. We give a negative answer using a randomized construction of bipartite posets whose linear discrepancy is asymptotic to the trivial upper bound $2 r-1$. For products of chains, we give alternative proofs of results obtained independently elsewhere.
\end{abstract}

\section{Introduction}

In seeking a linear extension to "fairly" represent a poset $P$, Tanenbaum, Trenk, and Fishburn [9] listed scenarios where one may want to keep incomparable elements of $P$ close together. For example, a doctor must treat waiting patients in a linear order, but the relation of "more urgent" is a partial order. Certainly $x$ should come before $y$ if $x$ is more urgent than $y$. When neither patient is more urgent, fairness suggests that neither should be treated a long time before the other.

A linear extension $L$ of $P$ is an order-preserving linear ordering of the elements of $P$; we write $L(x)$ for the position of $x$ in $L$. The uncertainty of $L$, written $t(L)$, is the maximum difference between the positions in $L$ of elements that are incomparable in $P$. By convention, $t(\mathbf{k})=0$, where $\mathbf{k}$ denotes a chain of $k$ elements. Finally, the linear discrepancy of $P$, written $\operatorname{ld}(P)$, is $\min \{t(L): L$ is a linear extension of $P\}$. The name reflects that $\operatorname{ld}(P)$ is a measure of how far $P$ is from a linear order.

*Gwangju Institute of Science and Technology, South Korea, jchoi351@gist.ac.kr

†West Virginia University, Morgantown WV, milans@math.wvu.edu

$\ddagger$ Zhejiang Normal University, Jinhua, China, and University of Illinois, Urbana IL, west@math.uiuc.edu. This research was partially supported by the National Security Agency under Award No. H98230-10-1-0363. 
For a fixed poset $P$, let $r$ denote the maximum number of elements incomparable to a given element (that is, the maximum vertex degree in the incomparability graph of $P$. Tanenbaum, Trenk, and Fishburn [9] suggested bounding $\operatorname{ld}(P)$ in terms of $r$, asking whether the inequality below always holds.

$$
\operatorname{ld}(P) \leq\left\lfloor\frac{3 r-1}{2}\right\rfloor
$$

The poset consisting of two disjoint $r$-element chains achieves equality in (1). The trivial upper bound is $\operatorname{ld}(P) \leq 2 r-1$ : for any two incomparable elements, every element between them on a linear extension is incomparable to at least one of them, so there are at most $2 r-2$ such elements. In particular, every linear extension has uncertainty at most $2 r-1$.

Rautenbach [8] proved (1) for $r \leq 3$ and improved the trivial general bound to $\operatorname{ld}(P) \leq$ $2 r-2$ (for $r \geq 2$ ). Keller and Young [6] proved (1) for posets whose order diagrams are disconnected, using a lemma that every poset contains an element whose deletion reduces the linear discrepancy by at most 1 . They also proved the stronger bound $\operatorname{ld}(P) \leq r$ when $P$ is an interval order.

We will prove (1) for posets of width 2. However, (1) does not hold for general posets; we give a randomized construction of bipartite posets whose linear discrepancy is asymptotic to the trivial upper bound $2 r-1$. These results suggest the following question:

Question 1.1. What is the largest linear discrepancy among posets of width $w$ in which every element is incomparable to at most $r$ other elements?

Linear discrepancy has also been studied for chain-products; write $\mathbf{k}^{d}$ for the product of $d$ chains that each have $k$ elements. For $k=2$, [9] proved $\operatorname{ld}\left(\mathbf{2}^{n}\right)=2^{n}-2^{(n+1) / 2}-1$ when $n$ is odd (and $n>1$ ), and $\operatorname{ld}\left(2^{n}\right)=2^{n}-3 \cdot 2^{n / 2}$ when $n$ is even. For products of two chains, we give a simpler proof of the result of Hong, Hyun, Kim, and Kim [5] that $\operatorname{ld}(\mathbf{m} \times \mathbf{n})=\lceil m n / 2\rceil-2($ when $\min \{m, n\} \geq 2$ and $m n \neq 4)$.

A general construction yields $\operatorname{ld}\left(\mathbf{k}^{d}\right) \leq\left(1-2^{-d+1}\right) k^{d}+O\left(k^{d-1}\right)$. We present unpublished proofs from Choi [3] that $\operatorname{ld}\left(\mathbf{k}^{3}\right)=\frac{3}{4} k^{3}-\frac{1}{2} k^{2}-1$ for even $k$ (also proved independently by Kim and Cheong [7]) and that $\operatorname{ld}\left(\mathbf{k}^{4}\right)=\frac{7}{8} k^{4}-\frac{3}{8} k^{3}-O\left(k^{2}\right)$ for even $k$. Independently, Cheong, Chae, and Kim [1] proved $\operatorname{ld}\left(\mathbf{k}^{3}\right)=\frac{3}{4} k^{3}-\frac{1}{2} k^{2}-\frac{1}{4} k$ for odd $k$. In [2], they extended the results to higher dimensions, showing that $\operatorname{ld}\left(\mathbf{k}^{d}\right)=\left(1-\frac{1}{2^{d-1}}\right) k^{d}-\frac{d-1}{2^{d-1}} k^{d-1}+O\left(k^{d-2}\right)$. They proved a more precise result when $k$ is odd: $\operatorname{ld}\left(\mathbf{k}^{d}\right)=k^{d}-k\left(\frac{k+1}{2}\right)^{d-1}+O(k)$.

\section{Bounds in terms of degree}

For an element $x$ in a poset $P$, let $I(x)$ denote the set of elements incomparable to $x$. Our first result is a sharp bound on $\operatorname{ld}(P)$ for posets of width 2 (as noted earlier, equality 
holds for the disjoint union of two $r$-element chains).

Theorem 2.1. If every element of a poset $P$ is incomparable to at most $r$ others, and $w(P)=2$, then $\operatorname{ld}(P) \leq\left\lfloor\frac{3 r-1}{2}\right\rfloor$.

Proof. We may assume $I(z) \neq \varnothing$ for all $z \in P$; if $I(z)=\varnothing$, then in any linear extension, all elements after $z$ are comparable to all elements before $z$, and deleting $z$ changes neither the linear discrepancy nor $r$.

By Dilworth's Theorem [4], $P$ is covered by disjoint chains $C$ and $D$. Let $C$ consist of $x_{1}, \ldots, x_{p}$, indexed in increasing order in $P$, and similarly let $D$ consist of $y_{1}, \ldots, y_{q}$. Each $I(z)$ is a nonempty interval on the chain $(C$ or $D)$ not containing $z$.

Define $a_{j}$ and $b_{j}$ by $I\left(y_{j}\right)=\left\{x_{a_{j}}, \ldots, x_{b_{j}}\right\}$. The $q$-tuples $a$ and $b$ are nondecreasing, and hence $\frac{a_{j+1}+b_{j+1}}{2} \geq \frac{a_{j}+b_{j}}{2}$ for $1 \leq j<q$. Form a linear extension $L$ of $P$ by inserting $y_{j}$ between $x_{s_{j}}$ and $x_{s_{j}+1}$ on $C$, where $s_{j}=\left\lfloor\frac{a_{j}+b_{j}}{2}\right\rfloor$.

Every incomparable pair consists of some $x_{i}$ and $y_{j}$ with $a_{j} \leq i \leq b_{j}$. Let $c_{i, j}$ and $d_{i, j}$ be the numbers of elements of $C$ and $D$ between $x_{i}$ and $y_{j}$ in $L$, so $\left|L\left(x_{i}\right)-L\left(y_{j}\right)\right|=c_{i, j}+d_{i, j}+1$. We claim that $c_{i, j}+d_{i, j} \leq \frac{3}{2}(r-1)=\frac{3 r-1}{2}-1$, which suffices.

Since $x_{i} \| y_{j}$, all elements of $D$ between $y_{j}$ and $x_{i}$ are incomparable to $x_{i}$, as is $y_{j}$, so $d_{i, j} \leq\left|I\left(x_{i}\right)\right| \leq r-1$. On the other hand, since $\left|I\left(y_{j}\right)\right| \leq r$ and $y_{j}$ is put at the middle of $I\left(y_{j}\right)$ along $C$, the number of elements of $I\left(y_{j}\right)$ between $y_{j}$ and any element incomparable to it is at most $\frac{1}{2}(r-1)$. Hence $m_{0}+m_{1} \leq \frac{3}{2}(r-1)$, as desired.

For large $r$, the value of $\operatorname{ld}(P)$ can be asymptotically close to the trivial upper bound. For this we give a probabilistic construction of bipartite posets (posets in which every element is maximal or minimal). When proving lower bounds for linear discrepancy, it is helpful to restrict the linear extensions that need to be considered.

Lemma 2.2. If $P$ is a bipartite poset, then $\operatorname{ld}(P)=t(L)$ for some linear extension $L$ in which all non-maximal elements precede all non-minimal elements.

Proof. If $L$ does not have this form, then some non-minimal element $y$ immediately precedes some non-maximal element $x$. Form $L^{\prime}$ from $L$ by switching $x$ and $y$. If $x$ is incomparable to a later element $z$, then also $y \| z$, so $L^{\prime}(z)-L^{\prime}(x)=L(z)-L(y)$ (similarly for $y$ incomparable to an earlier element $w)$. Thus $t\left(L^{\prime}\right) \leq t(L)$.

Lemma 2.2 is a special case of a lemma by Keller and Young [6], which takes somewhat more work. A pair $(x, y)$ of incomparable elements is critical if all elements below $x$ are below $y$ and all elements above $y$ are above $x$, so no added comparability can force $x$ above $y$. Lemma 4 of [6] states that for every poset, some linear extension with least uncertainty has $x$ before $y$ for every critical pair $(x, y)$ except those where $(y, x)$ also is critical.

Let $[n]$ denote $\{1, \ldots, n\}$. 
Theorem 2.3. When $r$ denotes the maximum degree of the incomparability graph, the bound $\operatorname{ld}(P) \leq 2 r-1$ is asympotically sharp, since such posets exist with $\operatorname{ld}(P) \geq 2 r-O(\sqrt{r \ln r})$.

Proof. Let $p$ be a function of $n$ taking values in $(0,1)$, tending to 0 as $n \rightarrow \infty$, but with $p n \rightarrow \infty$. Construct a bipartite poset $P_{n}$ with minimal elements $x_{1}, \ldots, x_{n}$ and maximal elements $y_{1}, \ldots, y_{n}$ by letting $x_{i}$ be less than $y_{j}$ in $P_{n}$ with probability $1-p$, for all $i, j \in$ $[n]$, independently. The expected number of elements incomparable to a given element is $(n-1)+p n$. By a standard computation, the probability that a given element is incomparable to more than $n-1+2 p n$ elements is bounded by $e^{-p n / 4}$. When multiplied by $2 n$, the bound still tends to 0 . Hence the probability of the event $r \leq n-1+2 p n$ tends to 1 .

In considering $\operatorname{ld}\left(P_{n}\right)$, by Lemma 2.2 we may confine our attention to extensions in which all $x_{i}$ precede all $y_{j}$. When $S$ and $T$ are sets of $m$ minimal and $m$ maximal elements, respectively, the probability that all of $S$ is under all of $T$ in $P_{n}$ is $(1-p)^{m^{2}}$. The probability that this holds for some $S$ and $T$ of size $m$ is at most $\left(\begin{array}{c}n \\ m\end{array}\right)^{2}(1-p)^{m^{2}}$. Since $\left(\begin{array}{c}n \\ m\end{array}\right)<\left(\frac{n e}{m}\right)^{m}$ and $(1-p) \leq e^{-p}$, this value is at most $\left(\frac{n e}{m}\right)^{2 m} e^{-p m^{2}}$. The bound tends to 0 when $m=\sqrt{n \ln n}$ and $p=3 \sqrt{(\ln n) / n}$. Thus with probability tending to 1 , in every linear extension some element among the first $\sqrt{n \ln n}$ is incomparable to some element among the last $\sqrt{n \ln n}$.

Thus $n-1 \leq r \leq n+6 \sqrt{n \ln n}$ and $\operatorname{ld}\left(P_{n}\right) \geq 2 n-2 \sqrt{n \ln n}$ for some instance of $P_{n}$ when $n$ is large. That is, $\operatorname{ld}(P)$ can be at least $2 r-O(\sqrt{r \ln r})$.

\section{Products of two chains}

We give a short proof of the result of $[5]$ that $\operatorname{ld}(\mathbf{m} \times \mathbf{n})=\lceil m n / 2\rceil-2$. We use another lemma by Keller and Young.

Lemma 3.1 (Lemma 3 of [6]). For any linear extension $L$ of a poset $P$, the maximum distance $t(L)$ is achieved only at critical pairs.

Proof. If $L(x)<L(y)$ and $(x, y)$ is not critical, then there is an element below $x$ but not $y$ or an element above $y$ but not $x$, and hence $t(L)>L(y)-L(x)$.

In $\mathbf{m} \times \mathbf{n}$, a pair $(x, y)$ of elements is a critical pair if and only if in one coordinate $x$ has value 1 and $y$ has the maximum ( $m$ or $n$ ), and in the other coordinate the value in $x$ exceeds that in $y$ by 1 . The lexicographic order on a set of $d$-tuples is defined by $x<y$ if $x$ is smaller in the first coordinate where $x$ and $y$ differ.

Example 3.2. When $m n$ is even, we first construct $L$ with $t(L)=m n / 2-1$. Assume by symmetry that $n$ is even, with $n=2 p$. Let $L^{\prime}$ put $\mathbf{m} \times \mathbf{p}$ in lexicographic order:

$$
(1,1)<\cdots<(1, p)<(2,1)<\cdots<(m-1, p)<(m, 1)<\cdots<(m, p) .
$$


Use $L^{\prime}$ for the first half of $L$. For the second half, use $L^{\prime}$ with $(0, p)$ added to each element. To compute $t(L)$, compare the positions within the critical pairs:

$$
\begin{array}{lll}
L(i, n)-L(i+1,1) & =m p-1 & \text { for } 1 \leq i<m, \\
L(m, j)-L(1, j+1) & =(m-1) p-1 & \text { for } j \in[n]-\{p\} \\
L(m, p)-L(1, p+1) & =-1 . &
\end{array}
$$

Thus $\operatorname{ld}(\mathbf{m} \times \mathbf{n}) \leq m p-1$.

Improving the upper bound by 1 will need a small change. It greatly increases small differences in order to reduce the difference by 1 for the critical pairs with largest difference.

Hong et al. [5] gave three separate constructions for the cases where $m$ and $n$ are both even, both odd, and of opposite parity. Our construction is simpler and has fewer cases. Their proof of the lower bound is by induction; we give a counting argument. Our proof incorporates ideas suggested by Daniel Cranston. (Note that $\operatorname{ld}(\mathbf{2} \times \mathbf{2})=1$.)

Theorem $3.3([5]) \cdot \operatorname{ld}(\mathbf{m} \times \mathbf{n})=\left\lceil\frac{m n}{2}\right\rceil-2$ when $m, n>1$ and $m n>4$.

Proof. Upper bound for mn even. By symmetry, assume that $n$ is even, with $n=2 p$. Modify the extension in Example 3.2 by moving $(1, p+1)$ from position $m p+1$ to position $p+1$. This increases $L(x)$ by 1 for $x \in([m]-\{1\}) \times[p]$. All other values remain the same. Now

$$
\begin{array}{lll}
L(i, n)-L(i+1,1) & =m p-2 & \text { for } 1 \leq i<m, \\
L(m, j)-L(1, j+1) & =(m-1) p & \text { for } 1 \leq j<n .
\end{array}
$$

Thus $\operatorname{ld}(\mathbf{m} \times \mathbf{n}) \leq m p-2$.

Upper bound for $m n$ odd. Number $\mathbf{m} \times \mathbf{n}$ by breaking the elements into five subgrids, each to be listed in lexicographic order as in (2) of Example 3.2. We describe the subgrids below, with formulas for the positions of elements in each. The order may be clearest from Figure 1, which shows the case $(m, n)=\{5,7\}$. The first two and last two subgrids leave the middle element of the grid in the middle position of the ordering. We express $L(i, j)$ as numbering from the beginning or the end or the middle value $\left\lceil\frac{m n}{2}\right\rceil$.

$\begin{array}{ccccl} & \text { subgrid } & \text { first } & \text { last } & L(i, j) \\ \text { I } & \left\lceil\left\lfloor\frac{m}{2}\right\rfloor\right] \times\left\lceil\left\lceil\frac{n}{2}\right\rceil\right] & (1,1) & \left(\left\lfloor\frac{m}{2}\right\rfloor,\left\lceil\frac{n}{2}\right\rceil\right) & (i-1)\left\lceil\frac{n}{2}\right\rceil+j \\ \text { II } & \left\{\left\lceil\frac{m}{2}\right\rceil, \ldots, m\right\} \times\left[\left\lfloor\frac{n}{2}\right\rfloor\right] & \left(\left\lceil\frac{m}{2}\right\rceil, 1\right) & \left(m,\left\lfloor\frac{n}{2}\right\rfloor\right) & \left\lceil\frac{m n}{2}\right\rceil-\left(\left\lfloor\frac{n}{2}\right\rfloor-j\right)-(m-i)\left\lfloor\frac{n}{2}\right\rfloor \\ \text { III } & \left(\left\lceil\frac{m}{2}\right\rceil,\left\lceil\frac{n}{2}\right\rceil\right) & \text { singleton } & \text { singleton } & \left\lceil\frac{m n}{2}\right\rceil \\ \text { IV } & \left\lceil\left\lceil\frac{m}{2}\right\rceil\right] \times\left\{\left\lceil\frac{n}{2}\right\rceil+1, \ldots, n\right\} & \left(1,1+\left\lceil\frac{n}{2}\right\rceil\right) & \left(\left\lceil\frac{m}{2}\right\rceil, n\right) & \left\lceil\frac{m n}{2}\right\rceil+(i-1)\left\lfloor\frac{n}{2}\right\rfloor+\left(j-\left\lceil\frac{n}{2}\right\rceil\right) \\ \text { V } & \left\{\left\lceil\frac{m}{2}\right\rceil+1, \ldots, m\right\} \times\left\{\left\lceil\frac{n}{2}\right\rceil, \ldots, n\right\} & \left(\left\lceil\frac{m}{2}\right\rceil+1,\left\lceil\frac{n}{2}\right\rceil\right) & (m, n) & m n-(n-j)-(m-i)\left\lceil\frac{n}{2}\right\rceil\end{array}$




\begin{tabular}{|ccc|cccc|}
\hline 15 & 16 & 17 & 32 & 33 & 34 & 35 \\
12 & 13 & 14 & 28 & 29 & 30 & 31 \\
\cline { 5 - 6 } 9 & 10 & 11 & 18 & 25 & 26 & 27 \\
& 6 & 7 & 8 & 22 & 23 & 24 \\
1 & 2 & 3 & 4 & 19 & 20 & 21 \\
\hline
\end{tabular}

Figure 1: Optimal discrepancy ordering for $[5] \times[7]$

By Lemma 3.1, it suffices to compute the difference in position for critical pairs. The desired bound $\lceil m n / 2\rceil-2$ follows from

$$
\begin{array}{lll}
L(i, n)-L(i+1,1) & =(m n-1) / 2-i & \text { for } 1 \leq i \leq\lfloor m / 2\rfloor, \\
L(i, n)-L(i+1,1) & =(m n-1) / 2-(m+1-i) & \text { for }\lceil m / 2\rceil \leq i \leq m, \\
L(m, j)-L(1, j+1) & =(m n-1) / 2-\lceil n / 2\rceil & \text { for } 1 \leq j \leq n-1 .
\end{array}
$$

Lower bound. We need $t(L) \geq(m n-4) / 2$ for every linear extension $L$. By symmetry, we may assume $L(m-1, n)=m n-1$. Either $L(2,1)=2$ or $L(1,2)=2$. If $L(1,2)=2$, then

$$
[L(m-1, n)-L(m, 1)]+[L(m, 1)-L(1,2)]=m n-3,
$$

and hence $t(L) \geq(m n-3) / 2$. Hence we may assume $L(2,1)=2$. If $L(1, n)>L(m, 1)$, then

$$
[L(m-1, n)-L(m, 1)]+[L(1, n)-L(2,1)] \geq m n-2,
$$

and hence $t(L) \geq(m n-2) / 2$. Thus we may assume $L(1, n)<L(m, 1)$.

Now let $a$ and $b$ be maximal such that $L(a, 1)<L(1,2)$ and $L(b, 1)<L(1, n)$. Similarly let $c$ and $d$ by minimal such that $L(c, n)>L(m, 1)$ and $L(d, n)>L(m, n-1)$. Note that $L(a, 1)=L(1,2)-1$ and $L(d, n)=L(m, n-1)+1$. Although $a=b$ and $c=d$ are possible, for clarity we show the case $a<b$ and $c<d$, where the elements are ordered as below:

$$
(a, 1),(1,2), \ldots,(b, 1), \ldots,(1, n), \ldots,(m, 1), \ldots,(c, n), \ldots,(m, n-1),(d, n) .
$$

Let $T=[L(m, 1)-L(1,2)]+[L(m, n-1)-L(1, n)]$. The definitions of $a$ and $d$ yield $T=L(m, 1)-(a+1)+m n-(m-d-1)-L(1, n)$. Since $(b+1,1), \ldots,(m-1,1)$ and $(2, n), \ldots,(c-1, n)$ all lie between $(1, n)$ and $(m, 1)$ (these sets are empty when $b=m-1$ or $c=2$, respectively), we have $L(m, 1)-L(1, n) \geq 1+m-1-b+c-2$. Thus

$$
T \geq m n-4+(d-b)+(c-a) .
$$


Regrouping yields $L(m, 1)-L(1,2)+a-c \geq \frac{m n-4}{2}$ or $L(m, n-1)-L(1, n)+b-d \geq \frac{m n-4}{2}$. Hence $t(L) \geq(m n-4) / 2$ unless $a>c$ and $b>d$. In that case $L(a-1, n)>L(m, 1)$ and $L(d+1,1)<L(1, n)$, yielding

$$
\begin{gathered}
L(a-1, n)-L(a, 1) \geq L(m, 1)+(a-c)-L(1,2)+1, \\
L(d, n)-L(d+1,1) \geq L(m, n-1)+1-L(1, n)+(b-d) .
\end{gathered}
$$

Summing yields $L(a-1, n)-L(a, 1)+L(d, n)-L(d+1, n) \geq T+(a-c)+(b-d)+2 \geq$ $m n-2$. Therefore, $\max \{L(a-1, n)-L(a, 1), L(d, n)-L(d+1,1)\} \geq \frac{m n-2}{2}$. We have proved $t(L) \geq \frac{m n}{2}-2$ in all cases.

\section{Upper Bound on $\operatorname{ld}\left(\mathbf{k}^{d}\right)$}

In this section we provide a constructive upper bound on $\operatorname{ld}\left(\mathbf{k}^{d}\right)$. In this and the next section we consider only even $k$. Since $(\mathbf{k}-\mathbf{1})^{d} \subseteq \mathbf{k}^{d} \subseteq(\mathbf{k}+\mathbf{1})^{d}$ and the top two terms in the upper and lower bounds for even $k$ match, this also yields asymptotic results for odd $k$, but more accurate results for odd $k$ appear in $[1,2]$. The lower bounds in the next section will show that our construction is optimal when $d=3$. A down-set (also called ideal) in a poset $P$ is a subset $D$ such that $x \in D$ and $y<x$ imply $y \in D$. An up-set (also called dual ideal) is a subset $U$ such that $x \in U$ and $y>x$ imply $y \in U$.

Lemma 4.1. Let $D$ be a down-set and $U$ an up-set in a poset $P$, both of size at least $r$. If $x<y$ for all $x \in D$ and $y \in U$, then $\operatorname{ld}(P) \leq|P|-r-1$.

Proof. Let $L_{1}, L_{2}, L_{3}$ be linear extensions of $D, P-D-U, U$, respectively. Concatenating $L_{1}, L_{2}, L_{3}$ yields a linear extension $L$ of $P$ with $t(L) \leq|P|-r-1$, since no element is incomparable to an element more than $|P|-r-1$ positions away from it in $L$.

Henceforth, let $l=k / 2$. Note that $\mathbf{k}^{d}$ is simply $[k]^{d}$ under the coordinatewise (product) ordering; the rank or height of an element $\left(x_{1}, \ldots, x_{d}\right)$ is $\left(\sum_{i=1}^{d} x_{i}\right)-d$. We focus on the bottom and top "orthants", but we also need certain other sets. Let $\Lambda=\{l+1, \ldots, k\}$.

$$
\begin{aligned}
A & =[l]^{d} \quad \text { and } \quad A^{\prime}=\{l+1, \ldots, k\}^{d}=\Lambda^{d} \\
\hat{A} & =\left\{x \in[k]^{d}: x_{d} \geq l+1 \text { and } x_{i} \leq l \text { for } i<d\right\}=[l]^{d-1} \times \Lambda \\
\hat{A}^{\prime} & =\left\{x \in[k]^{d}: x_{1} \leq l \text { and } x_{i} \geq l+1 \text { for } i>1\right\}=[l] \times \Lambda^{d-1} \\
B_{j} & =\left\{x \in[k]^{d}: x_{j}=l+1 \text { and } x_{i} \leq l \text { for } i \neq j\right\}=[l]^{j-1} \times\{l+1\} \times[l]^{d-j}, \\
B_{j}^{\prime} & =\left\{x \in[k]^{d}: x_{j}=l \text { and } x_{i} \geq l+1 \text { for } i \neq j\right\}=\Lambda^{j-1} \times\{l\} \times \Lambda^{d-j} .
\end{aligned}
$$


Each of $A, A^{\prime}, \hat{A}, \hat{A}^{\prime}$ has size $l^{d}$, while $B_{j}$ and $B_{j}^{\prime}$ have size $l^{d-1}$. Elements of "unprimed" sets have low rank in $\mathbf{k}^{d}$, while those of the "primed" sets have high rank. By definition,

(1) Every element of $A$ is below every element of $A^{\prime} \cup \bigcup_{j=2}^{d} B_{j}^{\prime}$.

(2) Every element of $A^{\prime}$ is above every element of $A \cup \bigcup_{j=1}^{d-1} B_{j}$.

(3) If $x \in B_{i}$ and $y \in B_{j}^{\prime}$, then $x \prec y$ if $i \neq j$ and $x \| y$ if $i=j$.

(4) If $d \geq 3$, then the sets $A, A^{\prime}, \hat{A}, \hat{A}^{\prime}, B_{1}, \ldots, B_{d-1}, B_{2}^{\prime}, \ldots, B_{d}^{\prime}$ are pairwise disjoint.

We ignore $B_{d}$ and $B_{1}^{\prime}$; they intersect $\hat{A}$ and $\hat{A}^{\prime}$, respectively. Note that $A$ is a down-set and $A^{\prime}$ is an up-set. All of $A$ is below all of $A^{\prime}$, and both have size $l^{d}$, so Lemma 4.1 yields $\operatorname{ld}\left(\mathbf{k}^{d}\right) \leq k^{d}-l^{d}-1$. This reduces the trivial bound $k^{d}-1$ by $l^{d}$. Careful use of elements near the bottom of $A$ and top of $A^{\prime}$ will permit subtracting more than twice as much.

Definition 4.2. Let $X=A \cup \hat{A} \cup \bigcup_{j=1}^{d-1} B_{j}$ and $X^{\prime}=A^{\prime} \cup \hat{A}^{\prime} \cup \bigcup_{j=2}^{d} B_{j}^{\prime}$. For $d \geq 3$ and $k$ even, the modified lex order on $[k]^{d}$ is a bijection $f:[k]^{d} \rightarrow\left\{1, \ldots, k^{d}\right\}$ defined as follows. Give the first $2 l^{d}+(d-1) l^{d-1}$ values to $X$ and the last $2 l^{d}+(d-1) l^{d-1}$ values to $X^{\prime}$. Within $X$, the sets $A, B_{1}, \ldots, B_{d-1}, \hat{A}$ appear in that order, with each of these $d+1$ sets listed in lexicographic order. Similarly, within $X^{\prime}$ the sets $\hat{A}^{\prime}, B_{2}^{\prime}, \ldots, B_{d}^{\prime}, A^{\prime}$ appear in that order, each listed in lexicographic order. The elements of $[k]^{d}-X-X^{\prime}$ receive the remaining (intermediate) positions in lexicographic order. See Figure 2.

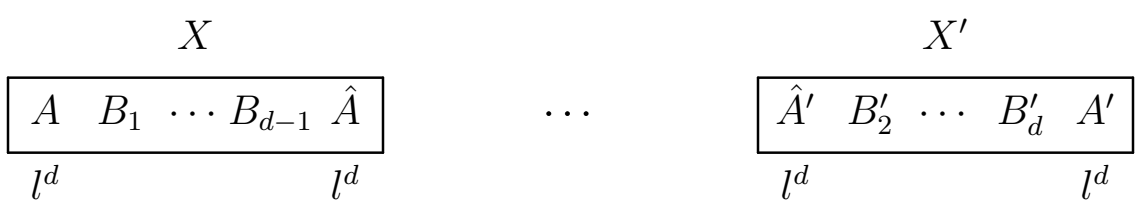

Figure 2: Sets in an ordering of $[k]^{d}$

We will show that $f$ defines a linear extension $L$ of $\mathbf{k}^{d}$ and compute $t(L)$. When $d=2$, the sets $\hat{A}$ and $\hat{A}^{\prime}$ are identical, and $B_{1} \cap B_{d}^{\prime}=\{(l+1, l)\} \neq \varnothing$, so in this case the numbering must be defined differently. Since Section 3 handles $\operatorname{ld}\left(\mathbf{k}^{2}\right)$, we assume $d \geq 3$.

Lemma 4.3. When $k$ is even and $d \geq 3$, the modifed lexicographic order $f$ in Definition 4.2 specifies a linear extension of $\mathbf{k}^{d}$.

Proof. Because the subsets we have ordered have been defined to be disjoint, and lexicographic order defines a bijection on any set of distinct $d$-tuples, the numbering $f$ is a bijection. If $x \prec y$ in $\mathbf{k}^{d}$, then $x$ comes before $y$ in the lexicographic order, so the lexicographic order on the elements of any subposet is a linear extension of that subposet. 
A numbering specifies a linear extension if and only if each initial segment of the resulting ordering is a down-set. By symmetry, it suffices to show that no element in any of $A, B_{1}, \ldots, B_{d-1}, \hat{A}$ is above any element that comes in a later set in the partition used to define $f$. First, $A$ is a down-set; no later element is less than an element of $A$.

Every element not in $A$ has some coordinate greater than $l$. For each element of $\bigcup_{j=1}^{d-1} B_{j}$, there is one such coordinate, and it has the minimum possible value, $l+1$. In particular, for $x \in B_{j}$ all elements below $x$ lie in $A \cup B_{j}$.

Finally, consider $x \in \hat{A}$. Every element below $x$ has value at most $l$ in each coordinate other than the last. Hence every such element is in $A \cup \hat{A}$.

Theorem 4.4. If $k$ is even and $d \geq 3$, then

$$
\operatorname{ld}\left(\mathbf{k}^{d}\right) \leq\left(1-\frac{1}{2^{d-1}}\right) k^{d}-\left(\frac{d-1}{2^{d-1}}\right) k^{d-1}-1 .
$$

Proof. With $l=k / 2$, by Lemma 4.3 it suffices to show that

$$
|f(x)-f(y)| \leq k^{d}-1-2 l^{d}-(d-1) l^{d-1}
$$

for any two incomparable elements $x$ and $y$, where $f$ is the modified lex order of Definition 4.2. Since $|X|=\left|X^{\prime}\right|=2 l^{d}+(d-1) l^{d-1}$, positions of elements in $[k]^{d}-X-X^{\prime}$ differ from both extremes by at most the desired bound. Hence we may ignore incomparable pairs containing elements outside $X \cup X^{\prime}$. Also, when comparing elements from $\hat{A}$ and $B_{j}^{\prime}$ or from $\hat{A}^{\prime}$ and $B_{j}$, there are $2 l^{d}+(d-1) l^{d-1}$ elements outside the pair (see Figure 2). Finally, in the poset all of $A$ is below all of $A^{\prime} \cup \bigcup_{j=2}^{d} B_{j}^{\prime}$ and all of $A^{\prime}$ is above all of $A \cup \bigcup_{j=1}^{d-1} B_{j}$. Hence it remains only to consider pairs $(x, y)$ of the following types:
(1) $x \in A$ and $y \in \hat{A}^{\prime}$,
(2) $x \in A^{\prime}$ and $y \in \hat{A}$,
(3) $x \in B_{i}$ and $y \in B_{j}^{\prime}$.

For type (3) we may assume that $i=j$, since otherwise $x \prec y$. All of $B_{j}$ is incomparable to all of $B_{j}^{\prime}$, so it suffices to compare the first element of $B_{j}$ with the last element of $B_{j}^{\prime}$, where $2 \leq j \leq d-1$. There are $l^{d}+(j-1) l^{d-1}$ elements before $B_{j}$ and $l^{d}+(d-j) l^{d-1}$ after $B_{j}^{\prime}$. Hence the reduction from $k^{d}-1$ in $|f(x)-f(y)|$ is $2 l^{d}+(d-1) l^{d-1}$, as desired.

Consider type (1): $x \in A$ and $y \in \hat{A}^{\prime}$; by symmetry, the argument also handles pairs of type (2). Since $x_{i} \leq l<l+1 \leq y_{i}$ for $2 \leq i \leq d$, incomparability requires $x_{1}>y_{1}$. In the lexicographic order on $A$ and on $\hat{A}^{\prime}$, differences in the first coordinate have the largest effect. It thus suffices to bound the difference when $x_{1}=y_{1}+1$, since $x \| y$. We may then also assume $x_{i}=1$ and $y_{i}=k$ for $i>1$.

Both $A$ and $\hat{A}^{\prime}$ have $l^{d-1}$ elements with each given value of the first coordinate. Hence $y_{1} l^{d-1}$ elements come before $x$ under $f$, and $\left|X^{\prime}\right|-y_{1} l^{d-1}$ elements come after $y$. Hence again the savings from $k^{d}-1$ in $|f(x)-f(y)|$ is $\left|X^{\prime}\right|$, which is $2 l^{d}+(d-1) l^{d-1}$, as desired. 


\section{Lower Bounds on $\operatorname{ld}\left(\mathbf{k}^{d}\right)$ for $d \in\{3,4\}$}

We show that optimal extensions of $\operatorname{ld}\left(\mathbf{k}^{d}\right)$ have a special form. Lower bounds for $d \in\{3,4\}$ result from studying extensions of this form more closely. The first part of the argument is valid for general chain products.

Consider $\prod_{j=1}^{d} \mathbf{k}_{j}$, consisting of $\left[k_{1}\right] \times \cdots \times\left[k_{d}\right]$ under the coordinate-wise ordering. We designate special elements of $\prod_{j=1}^{d} \mathbf{k}_{j}$. Element $z_{j}^{(i)}$ has value 1 in every coordinate except for $i$ in coordinate $j$. Element $\hat{z}_{j}^{(i)}$ has the maximum value in every coordinate except for $i$ in coordinate $j$. Note that $z_{j}^{(i)}<\hat{z}_{j^{\prime}}^{\left(i^{\prime}\right)}$ if $j \neq j^{\prime}$, and $z_{j}^{(i)} \| \hat{z}_{j^{\prime}}^{\left(i^{\prime}\right)}$ if $j=j^{\prime}$ and $i>i^{\prime}$.

We write $x_{j}$ for the value in coordinate $j$ of a $k$-tuple $x$, while $z_{j}^{(i)}$ denotes a particular element of $\prod\left[k_{i}\right]$. To avoid confusion, we will not use $z$ as a generic element. Let $A=$ $\left\{z_{j}^{(i)}: 1<i \leq k_{j}\right.$ and $\left.1 \leq j \leq d\right\}$, and let $\hat{A}=\left\{\hat{z}_{j}^{(i)}: 1 \leq i<k_{j}\right.$ and $\left.1 \leq j \leq d\right\}$. We call $A \cup \hat{A}$ the special elements of $\prod_{j=1}^{d} \mathbf{k}_{j}$. Here we omitted $z_{j}^{(1)}$ and $\hat{z}_{j}^{\left(k_{j}\right)}$, which are the bottom and top elements of the poset regardless of $j$. Disjointness of $A$ and $\hat{A}$ requires $d \geq 3$.

Given a linear extension $L$, we henceforth write $f(x)$ for $L(x)$, the position of an element in $L$. The term for such a function in [9] is linear labeling. We similarly write $f^{\prime}(x)$ for $L^{\prime}(x)$.

Lemma 5.1. If $L$ is a linear extension of $\prod_{j=1}^{d} \mathbf{k}_{j}$, then $t(L)$ equals $f\left(\hat{z}_{j}^{(i-1)}\right)-f\left(z_{j}^{(i)}\right)$ for some choice of $i$ and $j$.

Proof. The pairs of the form $\left(z_{j}^{(i)}, \hat{z}_{j}^{(i-1)}\right)$ are the critical pairs in $\prod_{j=1}^{d} \mathbf{k}_{j}$. Lemma 3.1 (Lemma 3 of [6]) applies.

Let $x$ and $y$ be incomparable elements of $A \cup \hat{A}$. A linear extension $L^{\prime}$ is an $x, y$-switch of an extension $L$ if (1) no element of $A \cup \hat{A}$ lies between $x$ and $y$ in $L$, and (2) $L^{\prime}$ agrees with $L$ except for reordering the interval from $x$ to $y$ so that $x$ and $y$ switch order.

Lemma 5.2. If a linear extension $L$ of $\prod_{j=1}^{k} \mathbf{k}_{j}$ has no elements of $A \cup \hat{A}$ between incomparable elements $x$ and $y$ of $A \cup \hat{A}$, then there is an $x, y$-switch of $L$.

Proof. Name $x$ and $y$ so that $f(x)<f(y)$. Let $D[y]=\{w: w \preceq y\}$ and $U[x]=\{w: w \succeq x\}$. Let $S=\{w: f(x) \leq f(w) \leq f(y)\}$.

We construct an extension $L^{\prime}$ that agrees with $L$ outside $S$. Within $S$, let $L^{\prime}$ put $D[y] \cap S$ first, then $S-D[y]-U[x]$, then $U[x] \cap S$. Within each of these sets, let the order in $L^{\prime}$ agree with the order in $L$. Since $D[y]$ is a down-set and $U[x]$ is an up-set, $L^{\prime}$ is a linear extension. Since $x$ and $y$ have switched order, and the elements not between them in $L$ have not moved, $L^{\prime}$ is an $x, y$-switch of $L$.

To obtain lower bounds, we compare positions of elements in critical pairs. It helps to restrict attention to extensions in a restricted class. For $a \in A$ with $a=z_{j}^{i}$, let $\hat{a}=\hat{z}_{j}^{(i-1)}$, 
and say that $a$ and $\hat{a}$ are in axis $j$ (translating the grid to have its lowest point at the origin would leave the elements of $A$ lying along the coordinate axes).

Lemma 5.3. Among linear extensions of $\prod_{j=1}^{d} \mathbf{k}_{j}$, uncertainty is minimized by an extension putting $\hat{a}$ later than a for all $a \in A$ and having the elements of $\hat{A}$ in the same order as the corresponding elements of $A$, except possibly for one fixed axis; some elements in this axis $j$ at the end of $A$ may appear after some elements in axis $j$ at the beginning of $\hat{A}$.

Proof. The first statement is a special case of Lemma 4 of [6] that there is an optimal extension reversing no critical pairs (unless the reversed pair is also critical). A direct proof here observes that $f(\hat{a})<f(a)$ prohibits elements of $A \cup \hat{A}$ between $\hat{a}$ and $a$ in $L$. Since $t(L)$ is achieved by some other (unreversed) critical pair, Lemma 5.2 enables the reversed critical pairs to be unreversed without increasing the uncertainty.

Next we want to put corresponding elements of $\hat{A}$ and $A$ in the same order without increasing the uncertainty. When two elements of $\hat{A}$ are out of order, we have $x, y, \hat{x}, \hat{y}$ with $f(x)<f(y)<f(\hat{y})<f(\hat{x})$; call this an inversion in $\hat{A}$. If no special elements lie between $\hat{y}$ and $\hat{x}$ in $L$, then Lemma 5.2 permits a $\hat{y}, \hat{x}$-switch. Let $L^{\prime}$ be the resulting extension. For $a \in A-\{x, y\}$, we have $f^{\prime}(\hat{a})-f^{\prime}(a)=f(\hat{a})-f(a)$. Also, $f^{\prime}(\hat{x})-f^{\prime}(x)<f(\hat{x})-f(x)$ and $f^{\prime}(\hat{y})-f^{\prime}(y)<f(\hat{x})-f(y)<f(\hat{x})-f(x)$. By Lemma 5.1, $t\left(L^{\prime}\right) \leq t(L)$.

If all of $A$ comes before all of $\hat{A}$, then switches as described above eliminate all inversions. Otherwise, since special elements from $A$ and $\hat{A}$ in different axes are comparable, all elements from $A \cup \hat{A}$ involved in pairs consisting of an element of $A$ appearing after an element of $\hat{A}$ lie in a single axis $j$. The order on them merges some initial portion of $\hat{z}_{j}^{(1)}, \ldots, \hat{z}_{j}^{\left(k_{j}-1\right)}$ with some terminal portion of $z_{j}^{(2)}, \ldots, z_{j}^{\left(k_{j}\right)}$, such that always $z_{j}^{(i)}$ comes before $\hat{z}_{j}^{(i)}$.

No element of $A$ in another axis follows $\hat{z}_{j}^{(1)}$. No element of $\hat{A}$ in another axis precedes $z_{j}^{\left(k_{j}\right)}$. Hence switches as described in the first part of the proof ensure that pairs in $\hat{A}$ not involving elements in axis $j$ appear in the same order as the corresponding elements in $A$.

The next definition and lemma about the computation of uncertainty do not require the restricted form obtained in Lemma 5.3. We consider any linear extension $L$ of $\prod_{j=1}^{d} \mathbf{k}_{j}$.

Definition 5.4. For $x \in A$, let $r(x)=\prod_{j=1}^{d} k_{j}-1-[f(\hat{x})-f(x)]$. Also, let $t_{j}(x)$ be the maximum $i$ such that $z_{j}^{(i)}$ precedes $x$ in $L$, and let $\hat{t}_{j}(x)$ be the maximum $i$ such that $\hat{z}_{j}^{\left(k_{j}+1-i\right)}$ follows $\hat{x}$ in $L$. Call $\left\{t_{j}(x)\right\}_{j=1}^{d}$ and $\left\{\hat{t}_{j}(x)\right\}_{j=1}^{d}$ the thresholds for $x$, and let $T(x)=$ $\prod_{j=1}^{d} t_{j}(x)+\prod_{j=1}^{d} \hat{t}_{j}(x)$. For $x, y \in A$, say that $y$ captures $x$ if $f(y)<f(x)<f(\hat{x})<f(\hat{y})$.

Note that each threshold for $x$ is at least 1 , and the number of elements outside the interval $[x, \hat{x}]$ in $L$ is $r(x)$. By Lemma $5.1, \operatorname{ld}\left(\prod_{j=1}^{d} \mathbf{k}^{d}\right)=\prod_{j=1}^{d} k_{j}-1-r(x)$ for some extension $L$ and $x \in A$. Hence we seek upper bounds on $r(x)$ for appropriately chosen $x$. 
Lemma 5.5. If $x \in A$, then $r(x) \leq T(x)$. Furthermore, if $x$ is in axis $j$, then $t_{j}(x)+\hat{t}_{j}(x)=$ $k_{j}$. Also $t_{j^{\prime}}(x)+\hat{t}_{j^{\prime}}(x) \leq k_{j^{\prime}}+1$ for $j^{\prime} \neq j$ if $x$ is not captured by an element in axis $j^{\prime}$.

Proof. The quantity $T(x)$ is the sum of the sizes of copies of the posets $\prod \mathbf{t}_{j}(x)$ and $\prod \hat{\mathbf{t}}_{j}(x)$ at the bottom and top of $\prod_{j=i}^{d} \mathbf{k}_{j}$, respectively. The lower product contains all $u$ such that $u_{j} \leq t_{j}(x)$ for all $j$. Any other element $v$ has $v_{j}>t_{j}(x)$ for some $j$. Changing the coordinates other than $v_{j}$ to 1 yields an element of $A$ that comes after $x$ and is less than $v$; hence $v$ is not before $x$. The symmetric argument holds for the top of $L$.

If $x=z_{j}^{(i)}$, then $t_{j}(x)=i-1$ and $\hat{t}_{j}(x)=k_{j}-(i-1)$. If $t_{j^{\prime}}(x)=i^{\prime}$, then let $y=z_{j^{\prime}}^{\left(i^{\prime}\right)}$. If $\hat{x}$ is not captured by $y$, then $f(\hat{y})<f(\hat{x})$, and hence $\hat{t}_{j^{\prime}}(x) \leq k_{j^{\prime}}-\left(i^{\prime}-1\right)$.

After Lemma 5.5, we have a bound on $T(x)$ of the form $\prod_{j=1}^{d} t_{j}+\left(k_{r}-t_{r}\right) \prod_{j \neq r}\left(k_{j}+1-t_{j}\right)$ when $x$ is in axis $r$ and is not captured. If we can choose $x$ so that each $t_{j}$ is near $k_{j} / 2$, then the resulting upper bound on $T(x)$ will be near $2^{-(d-1)} \prod_{j=1}^{d} k_{j}$, which would give the desired lower bound on $\operatorname{ld}\left(\prod_{j=1}^{d} k_{j}\right)$ asymptotically.

Optimizing the choice of $x$ for a particular extension and computing the resulting bound on $T(x)$ are both difficult when the chain sizes are distinct. Therefore, we henceforth assume that all chain sizes equal $k$. This restriction simplifies numerical arguments to prove that there is an optimal linear extension in which no special element is captured.

Theorem 5.6. Some linear extension of $\mathbf{k}^{d}$ with minimum uncertainty puts corresponding elements of $A$ and $\hat{A}$ in the same order; equivalently, no element of $A$ is captured.

Proof. Consider an extension $L$ with minimum uncertainty. By the argument in Lemma 5.3, it suffices to obtain an extension in which all of $\hat{A}$ appears after all of $A$, without increasing the uncertainty. If $L$ does not have this form, then by Lemma 5.3 there is one axis $j$ and integers $r$ and $s$ with $1 \leq r \leq k-1$ and $2 \leq s \leq k$ such that $\hat{z}_{j}^{(1)}, \ldots, \hat{z}_{j}^{(r)}$ is mixed with $z_{j}^{(s)}, \ldots, z_{j}^{(k)}$, following the other elements of $A$ and preceding the other elements of $\hat{A}$. The first of these elements is $\hat{z}_{j}^{(1)}$, and the last is $z_{j}^{(k)}$.

Let $S$ be this set of "in-between" elements. It suffices to obtain an extension $L^{\prime}$ such that (1) $L^{\prime}$ agrees with $L$ before the first element of $S$ and after the last element of $S$, (2) $L^{\prime}$ puts all elements of $S \cap A$ before all elements of $S \cap \hat{A}$, and (3) $t\left(L^{\prime}\right)=t(L)$.

It is easy to ensure (1) and (2). Since no element of $A$ is above any element of $\hat{A}$, the subposet on $S$ has an extension with $S \cap A$ before $S \cap \hat{A}$, and the remaining elements between the first and last elements of $S$ in $L$ can be inserted iteratively between the elements below them and the elements above them.

The problem is to ensure (3) for the resulting extension $L^{\prime}$. Possibly $f^{\prime}(\hat{a})-f^{\prime}(a)>$ $f(\hat{a})-f(a)$ for $a=z_{j}^{(i+1)}$ with $1 \leq i \leq \min \{r, s-1\}$. It suffices to produce an element $b \in A$ such that $f^{\prime}(\hat{a})-f^{\prime}(a)<f^{\prime}(\hat{b})-f^{\prime}(b)=f(\hat{b})-f(b)$. 
Let $b$ be the first element of $A$ in $L$ not on axis $j$; let $j^{\prime}$ be its axis. Note that $\hat{b}$ is the first element of $\hat{A}$ not in axis $j$, and $S$ is between $b$ and $\hat{b}$. Since $f^{\prime}(\hat{b})>f^{\prime}(\hat{a})$, the desired inequality holds unless $f(a)=f^{\prime}(a)<f^{\prime}(b)=f(b)$. It suffices to prove that $L^{\prime}$ has more elements between $\hat{a}$ and $\hat{b}$ than between $a$ and $b$.

Elements between $a$ and $b$ in $L$ and $L^{\prime}$ cannot be above any element of $A$ outside axis $j$ and hence exceed 1 only in position $j$. Thus they can only be $z_{j}^{(i+2)}, \ldots, z_{j}^{(s-1)}$, and there are at most $k-3$ of them. On the other hand, all elements of $\mathbf{k}^{d}$ between $z_{j}^{(k)}$ and $\hat{b}$ lie between $\hat{a}$ and $\hat{b}$ on both $L$ and $L^{\prime}$. Note that $\hat{b}$ and $z_{j}^{(k)}$ agree in positions $j$ and $j^{\prime}$ (with values $k$ and 1). In all other positions (at least one), $z_{j}^{(k)}$ has 1 , while $\hat{b}$ has $k$. The elements we are counting have any value between 1 and $k$ there, so there are at least $k-2$ of them.

The argument for elements of $S \cap \hat{A}$ is symmetric to this.

With optimal extensions restricted as in Lemma 5.6, the lower bounds for $\operatorname{ld}\left(\mathbf{k}^{3}\right)$ and $\operatorname{ld}\left(\mathbf{k}^{4}\right)$ are easy to prove. These simple arguments do not work for $d>4$, because the resulting bound on $T(x)$ for a single element $x$ is not tight enough. Proving sharp bounds for larger $d$ seems to require considering more elements on the extensions.

Theorem 5.7. $\operatorname{ld}\left(\mathbf{k}^{3}\right) \geq \frac{3}{4} k^{3}-\frac{1}{2} k^{2}-1$ when $k$ is even.

Proof. Let $m=k / 2$ (suggesting "middle"). By Lemma 5.6, it suffices to consider a linear extension with corresponding elements of $A$ and $\hat{A}$ in the same order and find an element $x \in A$ such that $r(x) \leq \frac{1}{4}\left(k^{3}+2 k^{2}\right)=2 m^{3}+2 m^{2}$.

Consider the following elements:

$$
\begin{gathered}
a=(m+1,1,1), b=(1, m+1,1), c=(1,1, m+1) \\
\hat{a}=(m, k, k), \hat{b}=(k, m, k), \hat{c}=(k, k, m) .
\end{gathered}
$$

By symmetry, we may assume that $f(a)<f(b)<f(c)$. By Lemma 5.5, it suffices to show that $T(b) \leq 2 m^{3}+2 m^{2}$.

The definition of $b$ yields $t_{2}(b)=\hat{t}_{2}(b)=m$. Since $b$ is uncaptured, $t_{1}(b)+\hat{t}_{1}(b) \leq k+1$ and $t_{3}(b)+\hat{t}_{3}(b) \leq k+1$, by Lemma 5.5. Let $t_{1}(b)=m+1+i$ and $t_{3}(b)=m-j$. Since $f(a)<f(b)<f(c)$, we have $t_{1}(b) \geq m+1$ and $t_{3}(b) \leq m$, so $i$ and $j$ are nonnegative. Now

$$
\begin{aligned}
T(b) & \leq(m+1+i) m(m-j)+(m-i) m(m+1+j) \\
& =2 m^{3}+2 m^{2}-2 i j-i-j \leq 2 m^{3}+2 m^{2} .
\end{aligned}
$$

The idea for $d=4$ is similar, but the increase in the number of coordinates requires a more careful choice of the key element $x$ before bounding $T(x)$.

Theorem 5.8. $\operatorname{ld}\left(\mathbf{k}^{4}\right) \geq \frac{7}{8} k^{4}-\frac{3}{8} k^{3}-\frac{1}{4} k^{2}-1$ when $k$ is even. 
Proof. Again let $m=k / 2$, and let $L$ be an extension without captured elements. By Lemma 5.5, it suffices to find $x \in A$ such that $T(x) \leq \frac{1}{8} k^{4}+\frac{3}{8} k^{3}+\frac{1}{4} k^{2}=2 m^{4}+3 m^{3}+m^{2}$.

Consider the following elements:

$$
\begin{gathered}
a=(m+1,1,1,1), b=(1, m+1,1,1), c=(1,1, m+1,1), d=(1,1,1, m+1) \\
\hat{a}=(m, k, k, k), \hat{b}=(k, m, k, k), \hat{c}=(k, k, m, k), \hat{d}=(k, k, k, m) .
\end{gathered}
$$

By symmetry, we may assume that $f(a)<f(b)<f(c)<f(d)$.

We choose a special element $x \in A$ such that $f(b) \leq f(x) \leq f(c)$. For all such $x$, let $\alpha(x)=t_{2}(x)+t_{3}(x)-k$; we seek $x$ such that $\alpha(x)=0$. Let $p$ and $q$ be the numbers of elements of $A$ in axes 2 and 3, respectively, in the part of $L$ strictly between $b$ and $c$. Since $t_{2}(b)=t_{3}(c)=m$, we have $\alpha(b)=-q$ and $\alpha(c)=p+1>0$. Also, $t_{2}+t_{3}$ increases by at most 1 with each step, increasing only when leaving an element in axis 2 or 3 . Let $x$ be the last element is in axis 2 or 3 such that $\alpha(x)=0$. (For example, $x=b$ when $q=0$, and $x$ is the last element in axis 2 or 3 before $c$ when $p=0$ and $q>0)$.

Since $\alpha(x)=0$, we have $t_{2}(x)=m+h$ and $t_{3}(x)=m-h$ for some nonnegative $h$. The resulting simple expression for $t_{2}(x) t_{3}(x)$ is the reason for choosing $x$ as we have. We bound $T(x)$. Since $t_{2}(x)=m+h$ and $t_{3}(x)=m-h$ and $x$ is uncaptured, Lemma 5.5 implies that $\hat{t}_{2}(x)=m-h$ and $\hat{t}_{3}(x) \leq m+1+h$ if $x$ is in axis 2 , but $\hat{t}_{3}(x)=m+h$ and $\hat{t}_{2}(x) \leq m+1-h$ if $x$ is in axis 3 . We keep the two cases together by writing $\hat{t}_{2}(x) \hat{t}_{3}(x)=(m-h)(m+1+h)$, where $0 \leq h \leq m$ if $x$ is in axis 2, but $0 \geq h \geq-m$ if $x$ is in axis 3 .

Also $t_{1}(x)+\hat{t}_{1}(x) \leq k+1$ and $t_{4}(x)+\hat{t}_{4}(x) \leq k+1$. Since $f(a)<f(x)$, we have $t_{1}=m+1+i$ with $0 \leq i<m$, and $f(x)<f(d)$ implies $t_{4}(x)=m-j$ with $0 \leq j \leq m$. Now

$$
T(x) \leq(m+1+i)\left(m^{2}-h^{2}\right)(m-j)+(m-i)(m-h)(m+1+h)(m+1+j) .
$$

To complete the proof, we maximize the upper bound over all choices of $h, i, j$. For fixed $h$ and $j$, the derivative with respect to $i$ is $\left(m^{2}-h^{2}\right)(-2 j-1)-(m-h)(m+1+j)$, which is negative regardless of the sign of $h$. Hence we set $i=0$. Now the derivative of $(m+1)\left(m^{2}-h^{2}\right)(m-j)+m(m-h)(m+1+h)(m+1+j)$ with respect to $j$ is $h^{2}-m h$, which has opposite sign to $h$. We set $j=0$ when $x$ is in axis 2 and $j=m$ when $x$ is in axis 3. Now the upper bound is $m(m+1)(m+h) 2(m+.5-h)$ with $0 \leq h \leq m$ or $m(m-h)(m+1+h)(2 m+1)$ with $0 \geq h \geq-m$. Each is maximized (for integers) when $h=0$, where they both equal $2 m^{4}+3 m^{3}+m^{2}$.

Finally, we combine the upper and lower bounds. When $k$ is even we have $\operatorname{ld}\left(\mathbf{k}^{d}\right) \leq$ $\frac{2^{d-1}-1}{2^{d-1}} k^{d}-\frac{d-1}{2^{d-1}} k^{d-1}-1$ from Theorem 4.4. Combining this with Theorem 5.7 and Theorem 5.8 yields the following statement. 
Corollary 5.9. When $k$ is even, $\operatorname{ld}\left(\mathbf{k}^{3}\right)=\frac{3}{4} k^{3}-\frac{1}{2} k^{2}-1$ and $\operatorname{ld}\left(\mathbf{k}^{4}\right)=\frac{7}{8} k^{4}-\frac{3}{8} k^{3}-O\left(k^{2}\right)$.

As remarked earlier, having the second term for even $k$ yields the asymptotic solution for all $k$.

Corollary 5.10. For all $k, \operatorname{ld}\left(\mathbf{k}^{3}\right)=\frac{3}{4} k^{3}+O\left(k^{2}\right)$ and $\operatorname{ld}\left(\mathbf{k}^{4}\right)=\frac{7}{8} k^{4}+O\left(k^{3}\right)$.

\section{Acknowledgements}

We thank the referees for a thorough reading and numerous useful suggestions.

\section{References}

[1] M. Cheong, G.-B. Chae, and S.-M. Kim, The linear discrepancy of the product of three chains of the same size, submitted.

[2] M. Cheong, G.-B. Chae, and S.-M. Kim, New bounds and an asymptotic result on the linear discrepancy of a product of chains, submitted.

[3] J. Choi, Ph.D. thesis, University of Illinois (2008).

[4] R. P. Dilworth, A decomposition theorem for partially ordered sets. Ann. of Math. (2) 51 (1950), 161-166.

[5] S. Hong, J. Hyun, H. Kim, and S. Kim, Linear discrepancy of the product of two chains, Order 22 (2005), 63-72.

[6] M. T. Keller and S. J. Young, Degree bounds for linear discrepancy of interval orders and disconnected posets. Discrete Math. 310 (2010), 2198-2203.

[7] S.-M. Kim and M. Cheong, The linear discrepancy of the product of three chains of size 2n. Far East J. Math. Sci. (FJMS) 30 (2008), 285-298.

[8] D. Rautenbach, A note on linear discrepancy and bandwidth. J. Combin. Math. Combin. Comput. 55 (2005), 199-208.

[9] P. Tanenbaum, A. Trenk, and P. Fishburn, Linear discrepancy and weak discrepancy of partially ordered sets, Order 18 (2001), 201-225. 